\title{
Synthesis and Characterization of Potential Impurities in Levothyroxine
}

\author{
DEEPAK PANMANAD*, MANDAR JOSHI, RAHUL PATIL, \\ PRIYANKA JOSHI and VIDYADHAR JADHAV
}

Prof. Dr. S. C. Bhattacharya Organic Synthesis Laboratory, VerGo Pharma Research Lab. Pvt. Ltd., Plot B5, Phase 1A, Verna Industrial Estate, Verna, Salcette, Goa-403722, India eepak.panmand@vergopharma.com

Received 16 June 2016 / Accepted 26 June 2016

\begin{abstract}
Five potential USP grade levothyroxine impurities i.e. $N$-methyl amide (2), T4-amine $O$ methyl (7), T4-acetamide (9), $N$-acyl-T4 (10) and $N$-formyl-T4 (11) have been synthesized. Each Levothyroxine impurity was characterized based on their spectral data (IR, ${ }^{1} \mathrm{H}$ NMR and Mass). The synthesis and characterisation of the Levothyroxine impurities are discussed.
\end{abstract}

Keywords: Levothyroxine, Impurity, Synthesis, Characterization, Decarboxylation

\section{Introduction}

Levothyroxine or $L$-thyroxine is an endogenous hormone secreted by the thyroid gland, synthetic form of thyroxine (T4). Levothyroxine is used to treat thyroid hormone deficiency and treatment of goiter ${ }^{1}$. It is also used to prevent the recurrence of thyroid cancer. Levothyroxine sodium is an active salt of levothyroxine, sold as drug under different brand name such as synthroid, levoxyl, levothroid, unithroid and tirosint.

Levothyroxine $\mathbf{1}$ chemically known as (S)-2-amino-3-[4-(4-hydroxy-3,5diiodophenoxy)-3,5-diiodophenyl]propanoic acid can be synthesized by various methods. Chalmers et al., described multi-step process ${ }^{2}$ involves $L$-tyrosine nitration followed by $N$-acetylation and then esterification to give 3,5-dinitro- $N$-acetyl- $L$-tyrosine ethyl ester. The resulting compound underwent coupling with $p$-methoxyphenol to give the corresponding diphenylether which on hydrogenation results in diamine. Diamine compound on iodination via diazotization, demethylation and hydrolysis, finally ring iodination to give Levothyroxine as shown in Scheme 1. Later development of levothyroxine synthesis involves iodination of 3,5-diodothyronine ${ }^{3}$ and coupling between 3,5-diiodo $L$-tyrosine copper complex and bis( $p$-anisyl)iodonium iodide ${ }^{4}$. Levothyroxine synthesis development is well documented as reviewed by Reddy et al.. ${ }^{5}$. There was continuous improvement in levothyroxine synthesis not only to develop simple, convenient, economical and commercially feasible process but to avoid or minimize the level of any possible impurity. 
事<smiles>COC(=O)C(Cc1cc([N+](=O)[O-])c(O)c([N+](=O)[O-])c1)NC(Cc1cc(N=O)c(Oc2ccc(OC)cc2[N+](=O)[O-])c([N+](=O)[O-])c1)C(=O)OC</smiles>

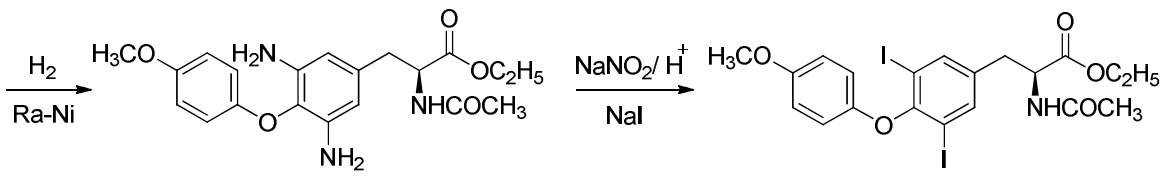<smiles>CC(C)OC(=O)NCCN</smiles>

Scheme 1. Synthesis of Levothyroxine (1)

Related substances or impurities in the form of by-products or process impurities are possible during the manufacturing process or storage of the drug substance ${ }^{6}$. The acceptance criteria for these impurities are stringent and based on the guidelines laid down by the International Conference on Harmonization (ICH) and pharmacopeia. As per the stringent regulatory requirements recommended by $\mathrm{ICH}$, the impurities $\geq 0.1 \%$ must be identified and characterized. Furthermore, it is equally important to have impurity in pure form required for analytical development such as specificity, linearity, accuracy, limit of detection (LOD), limit of quantification (LOQ), robustness and relative retention factor. Easy way to get impurity in pure form is to degrade API product and enrich desired impurity followed by isolation by column or flash chromatography or PREP-HPLC purification depending upon level of impurity present in degraded API. But if impurity is not forming at sufficient level by various degradation methods or if it is process related then there is no choice but to plan for synthesis of particular impurity.

A literature survey revealed that nobody has reported synthesis of levothyroxine impurities as mention below in Figure 1, except $N$-acyl-T4 10 in the purified form. The present communication involves the synthesis of impurities from levothyroxine $\mathbf{1}$ or key raw material and characterized by spectroscopic techniques and HPLC RRT.

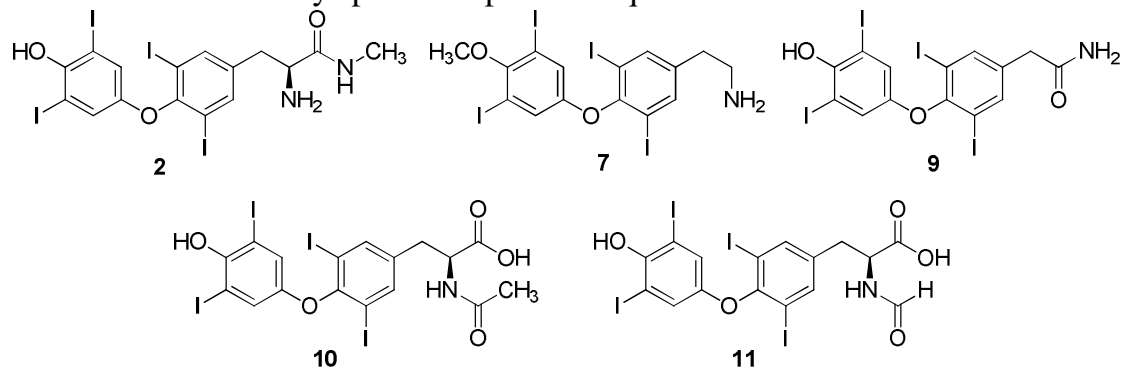

Figure 1. Structures of levothyroxine impurity as per USP 


\section{Experimental}

All moisture and air sensitive reactions were carried out under nitrogen atmosphere. The solvents were dried using standard methods, distilled and stored under nitrogen. Reactions were monitored by Thin layer chromatography (TLC) were run on silica gel 60 F254 precoated plates $(0.25 \mathrm{~mm}$, Merck, Art. 5554) and spots were visualized inside an UV cabinet under short UV. Infrared spectra were recorded on IR Affinity-1, Shimadzu. ${ }^{1} \mathrm{H}$ NMR spectra were recorded on Bruker Advance III $400 \mathrm{MHz}$ with TMS as an internal standard. Mass spectra were obtained using LC-MS API-2000, ABSciex. HPLC analysis done as mentioned in USP monograph using column $4.6 \mathrm{~mm} \times 15 \mathrm{~cm} ; 5 \mu \mathrm{m}$ packing L7 and 4.0 $\mathrm{mm} \times 15 \mathrm{~cm}$; 3-m packing L1. All reagents were purchased from Aldrich (India) and S. D. Fine Chemicals, Mumbai. 2-(4-(4-Hydroxy-3,5-diiodophenoxy)-3,5-diiodophenyl)acetic acid 8 was purchased from Aldrich (India). All reactions were carried out in purified solvents. Compound $N$-Boc Levothyroxine (5) was synthesized as per literature procedure ${ }^{11}$.

\section{(S)-2-Amino-3-(4-(4-hydroxy-3,5-diiodophenoxy)-3,5-diiodophenyl)- $N$ - methylpropanamide (2)}

In a $100 \mathrm{~mL}$ two-necked flask levothyroxine $(1.0 \mathrm{~g}, 1.29 \mathrm{mmol})$ and methanol $(30 \mathrm{~mL})$ were added under nitrogen atmosphere. Thionyl chloride $(1.63 \mathrm{~mL}, 22.47 \mathrm{mmol})$ was added drop wise at $0-5{ }^{\circ} \mathrm{C}$, the reaction mass was refluxed over a period of $2 \mathrm{~h}$. Then, concentrated under reduced pressure to obtain light yellow intermediate in crude form, which was dissolved in dropwise addition of saturated methanolic solution of methylamine $(20 \mathrm{~mL})$ and stirred at room temperature for $20 \mathrm{~h}$. After complete conversion, as monitored by TLC (Mobile phase: $\mathrm{CH}_{2} \mathrm{Cl}_{2-} \mathrm{CH}_{3} \mathrm{OH}, 95: 5 \mathrm{v} / \mathrm{v}$ ), the reaction mass was diluted with water (70 $\mathrm{mL})$ and extracted with dichloromethane $(3 \times 70 \mathrm{~mL})$, organic layers were combined, dried over anhydrous sodium sulfate and concentrated in vacuum to afford crude product. A purification by flash column chromatography on silica gel (100-200 mesh) in $\mathrm{CH}_{2} \mathrm{Cl}_{2}$ $\mathrm{CH}_{3} \mathrm{OH}$ gradient to obtain light yellow solid with yield of $(0.78 \mathrm{~g}) 76 \%$. Melting Point: $110.7{ }^{\circ} \mathrm{C}$. HPLC Purity: $94.38 \%$. IR $\left(\mathrm{cm}^{-1}\right): 3686,2938,1643,1427,1418,1234,1161$, 1053, 1009, 901, 831, 704, 696. ${ }^{1} \mathrm{H}$ NMR $\left(\mathrm{CDCl}_{3}\right): \delta 7.64(\mathrm{~s}, 2 \mathrm{H}, \mathrm{ArH}), 7.05(\mathrm{~s}, 2 \mathrm{H}, \mathrm{ArH})$, 3.49-3.57 (dd, $J=8.8$ and $4.0 \mathrm{~Hz}, 1 \mathrm{H}, \mathrm{CH}), 3.11-3.16(\mathrm{dd}, J=13.6 \mathrm{~Hz} \& 4.0 \mathrm{~Hz}, 1 \mathrm{H}, \mathrm{CH})$, 2.73-2.79 (d, $\left.J=4.8 \mathrm{~Hz}, 3 \mathrm{H}, \mathrm{CH}_{3}\right), 2.53-2.63(\mathrm{dd}, J=14.4 \& 9.2 \mathrm{~Hz}, 1 \mathrm{H}, \mathrm{CH})$. Mass spectrum $\mathrm{m} / \mathrm{z} 790.6[\mathrm{MH}]^{+}, 788.4[\mathrm{M}-\mathrm{H}]^{-}$.

\section{(S)-Methyl-2-((tert-butoxycarbonyl)amino)-3-(4-(3,5-diiodo-4-methoxyphenoxy)- 3,5-diiodo-phenyl)propionates (4)}

In a $100 \mathrm{~mL}$ two-necked flask $N$-Boc levothyroxine $(5.0 \mathrm{~g}, 5.70 \mathrm{mmol})$ and $\mathrm{N}, \mathrm{N}$ dimethylformamide $(35 \mathrm{~mL})$ were introduced. Then, sodium hydride $(0.46 \mathrm{~g} ; 19.38 \mathrm{mmol})$ was added at $0{ }^{\circ} \mathrm{C}$ in lot wise and stirred reaction over $1.5 \mathrm{~h}$. Reaction mass cooled to $0{ }^{\circ} \mathrm{C}$, methyl iodide $(0.24 \mathrm{~mL} ; 13.11 \mathrm{mmol})$ was added dropwise and maintained for $5 \mathrm{~h}$ at room temperature. Reaction progress was monitored by TLC TLC (Mobile phase: $\mathrm{CH}_{2} \mathrm{Cl}_{2}$ $\left.\mathrm{CH}_{3} \mathrm{OH}, 95: 5 \mathrm{v} / \mathrm{v}\right)$. Reaction mass was quenched with addition of cold dropwise water $(30 \mathrm{~mL})$, filtered to obtain off white solid with $(5.05 \mathrm{~g} ; 5.60 \mathrm{mmol})$ yield of $98.05 \%$. Melting Point: $90{ }^{\circ} \mathrm{C}$. IR $\left(\mathrm{cm}^{-1}\right): 3366,3354,1651,1437,1386,1234,1165,993,847 .{ }^{1} \mathrm{H}$ NMR $\left(\mathrm{CDCl}_{3}\right): \delta 7.64(\mathrm{~s}$, 2H, ArH), 7.15 (s, 2H, ArH), 5.10-5.17 (d, $J=6.0 \mathrm{~Hz}, 1 \mathrm{H}, \mathrm{NH}), 4.51-4.59$ (dd, $J=13.1 \&$ $6.7 \mathrm{~Hz}, 1 \mathrm{H}, \mathrm{CH}), 3.84\left(\mathrm{~s}, 3 \mathrm{H}, \mathrm{CH}_{3}\right), 3.76\left(\mathrm{~s}, 3 \mathrm{H}, \mathrm{CH}_{3}\right), 3.05-3.29(\mathrm{dd}, J=14.0$ \& $4.8 \mathrm{~Hz}, 1 \mathrm{H}$, $\mathrm{CH}), 2.96-301(\mathrm{dd}, J=14.4 \& 6.0 \mathrm{~Hz}, 1 \mathrm{H}, \mathrm{CH}), 1.46\left(\mathrm{~s}, 9 \mathrm{H}, \mathrm{CH}_{3}\right)$. Mass spectrum $\mathrm{m} / z .928 .3$ $[\mathrm{M}+\mathrm{Na}]^{+}$. 
(S)-2-Amino-3-(4-(3,5-diiodo-4-methoxyphenoxy)-3,5-diiodophenyl)propanoic acid (5)

To a solution of $O$-methyl levothyroxine methyl ester $(4.8 \mathrm{~g}, 5.30 \mathrm{mmol})$ in 1,4-dioxane $(6.64 \mathrm{~mL}) 2 \mathrm{~N}$ sodium hydroxide solution $(48 \mathrm{~mL}, 10$ volume) was added and stirred at room temperature for $5 \mathrm{~h}$. After completed conversion, as monitored by TLC (Mobile phase: $\mathrm{CH}_{2} \mathrm{Cl}_{2}-\mathrm{CH}_{3} \mathrm{OH}, 90: 10 \mathrm{v} / \mathrm{v}$ ), water was added and filtered to obtain solid (3.91 g; 4.38 mmol; 82\%), Melting Point: $107.5^{\circ} \mathrm{C}$. IR $\left(\mathrm{cm}^{-1}\right): 3348,2966,2936,2924,1680,1582,1526$, 1435, 1365, 1332, 1016, 709. ${ }^{1} \mathrm{H}$ NMR $\left(\mathrm{CDCl}_{3}\right): \delta 7.64$ (s, 2H, ArH), $7.10(\mathrm{~s}, 2 \mathrm{H}, \mathrm{ArH})$, $5.10-5.13(\mathrm{~d}, J=7.2 \mathrm{~Hz}, 1 \mathrm{H}, \mathrm{NH}), 4.50-4.52(\mathrm{~d}, J=6.8 \mathrm{~Hz}, 1 \mathrm{H}, \mathrm{CH}), 3.76\left(\mathrm{~s}, 3 \mathrm{H}, \mathrm{OCH}_{3}\right)$, $3.10-3.30$ (dd, $J=14.0 \& 5.2,1 \mathrm{H}, \mathrm{CH}), 2.85-2.99(\mathrm{dd}, J=14.0 \& 6.8,1 \mathrm{H}, \mathrm{CH}), 1.45(\mathrm{~s}, 9 \mathrm{H}$, $\left.\left(\mathrm{CH}_{3}\right)_{2}\right)$. Mass spectrum $m / z: 890.2[\mathrm{M}-\mathrm{H}]^{-}$.

(S)-2-amino-3-(4-(3,5-diiodo-4-methoxyphenoxy)-3,5-diiodophenyl)propanoic acid (6)

In a $100 \mathrm{~mL}$ two-necked flask $\mathrm{N}$-BOC-O-methyl levothyroxine $(3.92 \mathrm{~g}, 4.4 \mathrm{mmol})$ were introduced and cooled to $0{ }^{\circ} \mathrm{C}$. Trifluoroacetic acid ( $32 \mathrm{~mL}, 8$ volume) was added and stirred over $30 \mathrm{~min}$ at room temperature. After complete conversion, as monitored by TLC (Mobile phase: $\left.\mathrm{CH}_{2} \mathrm{Cl}_{2}-\mathrm{CH}_{3} \mathrm{OH}, 80: 20 \mathrm{v} / \mathrm{v}\right)$, ice cold water $(40 \mathrm{~mL})$ was added and filtered to obtain white solid, 3.0 g. (3.79 mmol, 87\%), Melting point: $125.1{ }^{\circ} \mathrm{C}$; IR $\left(\mathrm{cm}^{-1}\right): 3705,2949,1601$, $1581,1458,1435,1234,1053,995,914,770,708,663 .{ }^{1} \mathrm{H}$ NMR (DMSO- $\left.d_{6}\right): \delta 7.84(\mathrm{~s}, 1 \mathrm{H}$, $\mathrm{ArH}), 7.67(\mathrm{~s}, 1 \mathrm{H}, \mathrm{ArH}), 7.21(\mathrm{~s}, 1 \mathrm{H}, \mathrm{ArH}), 7.13(\mathrm{~s}, 1 \mathrm{H}, \mathrm{ArH}), 3.72\left(\mathrm{~s}, 3 \mathrm{H}, \mathrm{CH}_{3}\right), 3.46-3.48$ $(\mathrm{t}, J=7.6 \& 4.0 \mathrm{~Hz}, 1 \mathrm{H}, \mathrm{CH}), 3.05-3.17(\mathrm{dd}, J=14.0 \& 9.2,1 \mathrm{H}, \mathrm{CH}), 2.76-2.84(\mathrm{dd}, J=$ $14.0 \& 9.2,1 \mathrm{H}, \mathrm{CH}), 3.01(\mathrm{bs}, 1 \mathrm{H}, \mathrm{CH})$. Mass spectrum $m / z: 789.7[\mathrm{M}-\mathrm{H}]^{-}$.

\section{2-(4-(3,5-Diiodo-4-methoxyphenoxy)-3,5-diiodophenyl)ethanamine (7)}

In a $100 \mathrm{~mL}$ two-necked flask $O$-methyl levothyroxine acid $(1.28 \mathrm{~g}, 1.61 \mathrm{mmol})$ in cyclohexanol $(4 \mathrm{~mL})$ were introduced and 2-cyclohexen-1-one $(0.139 \mathrm{~mL}, 0.14 \mathrm{mmol})$ was added and heated reaction at $150{ }^{\circ} \mathrm{C}$ for $40 \mathrm{~min}$. After completed conversion, as monitored by TLC (Mobile phase: $\mathrm{CH}_{2} \mathrm{Cl}_{2}-\mathrm{CH}_{3} \mathrm{OH}, 90: 10 \mathrm{v} / \mathrm{v}$ ), reaction mass was diluted in water $(30 \mathrm{~mL})$ and extracted in ethyl acetate, separated organic layers dried over sodium sulfate, concentrated to obtain crude mass which was purified by flash column chromatography using $\mathrm{CH}_{2} \mathrm{Cl}_{2}-\mathrm{CH}_{3} \mathrm{OH}$ 93:7 v/v gradient to obtain white solid $0.65 \mathrm{~g}(0.87 \mathrm{mmol}, 54 \%)$. Melting Point: $185{ }^{\circ} \mathrm{C}$. HPLC Purity: 96.59\%. IR $\left(\mathrm{cm}^{-1}\right)$ : 3057, 1678, 1435, 1408, 1204, 1142, 723. ${ }^{1} \mathrm{H}-\mathrm{NMR}\left(\mathrm{CDCl}_{3}\right): \delta 7.71(\mathrm{~s}, 2 \mathrm{H}, \mathrm{ArH}), 7.17(\mathrm{~s}, 2 \mathrm{H}, \mathrm{ArH}), 3.84\left(\mathrm{~s}, 3 \mathrm{H}, \mathrm{OCH}_{3}\right)$, 3.01-3.8 (t, $J=6.8 \mathrm{~Hz}, 2 \mathrm{H}), 2.72-2.78(\mathrm{t}, J=7.2 \& 6.8 \mathrm{~Hz}, 2 \mathrm{H})$. Mass spectrum $m / z: 747.3$ $[\mathrm{MH}]^{+}, 746.7[\mathrm{M}-\mathrm{H}]^{-}$.

\section{2-(4-(4-Hydroxy-3,5-diiodophenoxy)-3,5-diiodophenyl)acetamide (9)}

Thionyl chloride $(6.2 \mathrm{~mL}, 85.8 \mathrm{mmol})$ was added to a solution of T4-acetic acid $(0.300 \mathrm{~g}$; $0.40 \mathrm{mmol}$ ), reaction mixture was heated to reflux and stirred for $2 \mathrm{~h}$. Reaction was monitored by TLC $\left(\mathrm{CH}_{2} \mathrm{Cl}_{2}-\mathrm{CH}_{3} \mathrm{OH}, 95 / 5 \mathrm{v} / \mathrm{v}\right)$, after completion, concentrated under nitrogen atmosphere. Ammonium hydroxide was added and the solution stirred for $15 \mathrm{~min}$. The reaction mixture was diluted chloroform $(15 \mathrm{~mL})$ and washed with saturated sodium bicarbonate solution $(15 \mathrm{~mL})$, brine $(15 \mathrm{~mL})$, dried over sodium sulfate, concentrated under vacuum and purified by flash column using $\mathrm{CH}_{2} \mathrm{Cl}_{2}-\mathrm{CH}_{3} \mathrm{OH}$ gradient. Required fraction was concentrated and washed with diethyl ether to obtain pure product $0.240 \mathrm{~g}(0.30 \mathrm{mmol}$, 80\%). Melting Point: $160{ }^{\circ} \mathrm{C}$. HPLC Purity: 95.21\%. IR $\left(\mathrm{cm}^{-1}\right): 3400,1654,1416,1229$, 1180, 706, ${ }^{1} \mathrm{H}$ NMR (DMSO- $\left.d_{6}\right): \delta 9.30$ (bs, 1H, OH), 7.82 (s, 2H, ArH), 7.08 (s, 2H, ArH), $3.39\left(\mathrm{~s}, 2 \mathrm{H}, \mathrm{CH}_{2}\right)$. Mass spectrum $m / z: 769.4[\mathrm{M}+\mathrm{H}]^{+}$. 


\section{(S)-2-Acetamido-3-(4-(4-hydroxy-3,5-diiodophenoxy)-3,5-diiodophenyl)-propanoic} acid (10)

Reaction mixture of levothyroxine API $(1.0 \mathrm{~g}, 1.3 \mathrm{mmol})$ in acetic anhydride $5 \mathrm{~mL}$ (5 volume) was stirred at $139{ }^{\circ} \mathrm{C}$ for $1.5 \mathrm{~h}$. Reaction progress was monitored by $\mathrm{TLC}\left(\mathrm{CH}_{2} \mathrm{Cl}_{2}-\mathrm{CH}_{3} \mathrm{OH}\right.$, 9:1 v/v) and after completion, reaction mass cooled at room temperature and filtered to get crude product, which was purified by using flash column chromatography in $\left(\mathrm{CH}_{2} \mathrm{Cl}_{2}-\mathrm{CH}_{3} \mathrm{OH}-\right.$ TEA) mobile phase. Solid was triturated in $\mathrm{CH}_{3} \mathrm{OH}-\mathrm{Et}_{2} \mathrm{O}$ gives $0.70 \mathrm{~g}(0.85 \mathrm{mmol}, 66.66 \%)$. HPLC Purity: $92.83 \%$. Melting Point: $165.0^{\circ} \mathrm{C}$. IR $\left(\mathrm{cm}^{-1}\right): 3142,3131,1574,1533,1433$ 1393, 1233, 1132, 916, 707, 694. ${ }^{1} \mathrm{H}$ NMR (DMSO- $\left.d_{6}\right) \delta 7.71(\mathrm{~S}, 2 \mathrm{H}, \mathrm{ArH}), 7.61-7.67$ (d, $J=$ $7.2 \mathrm{~Hz}, 1 \mathrm{H}, \mathrm{NH}), 7.02(\mathrm{~s}, 2 \mathrm{H}, \mathrm{ArH}), 4.08-4.15(\mathrm{dd}, J=13.2 \& 4.8 \mathrm{~Hz}, 1 \mathrm{H}, \mathrm{CH}), 3.00-3.07$ (dd, $J=13.2 \& 4.8 \mathrm{~Hz}, 1 \mathrm{H}, \mathrm{CH}), 2.79-2.71(\mathrm{dd}, J=13.2 \& 8.0 \mathrm{~Hz}, 1 \mathrm{H}, \mathrm{CH}), 1.79\left(\mathrm{~S}, 3 \mathrm{H}, \mathrm{CH}_{3}\right)$. Mass spectrum $m / z: 819[\mathrm{M}+\mathrm{H}]^{+}, 841[\mathrm{M}+\mathrm{Na}]^{+}, 857[\mathrm{M}+\mathrm{K}]^{+}$.

\section{(S)-2-Formamido-3-(4-(4-hydroxy-3,5-diiodophenoxy)-3,5-diiodophenyl)-propanoic} acid (11)

Acetic anhydride $(1.9 \mathrm{~g} ; 18.5 \mathrm{mmol})$ was added dropwise to a solution of levothyroxine API $(2.0 \mathrm{~g}, 2.60 \mathrm{mmol})$ in formic acid $5 \mathrm{~mL}$ at $0{ }^{\circ} \mathrm{C}$, maintained for $1 \mathrm{~h}$ at $0-4{ }^{\circ} \mathrm{C}$. After completed conversion, as monitored by TLC (Mobile phase: $\mathrm{CH}_{2} \mathrm{Cl}_{2}-\mathrm{CH}_{3} \mathrm{OH}, 90: 10 \mathrm{v} / \mathrm{v}$ ), reaction mass was stirred for $1 \mathrm{~h}$ at room temperature. Ice cold water $(20 \mathrm{~mL})$ was added, solid obtained, filtered and triturated in diethyl ether to obtain pure product as white solid $1.1 \mathrm{~g}(1.38 \mathrm{mmol}, 56 \%)$. HPLC Purity: $94.09 \%$. Melting Point: $242.2^{\circ} \mathrm{C}$. IR $\left(\mathrm{cm}^{-1}\right): 3379$, 2953, 2922, 2853, 1705, 1586, 1454, 1433, 1242, 1178, 918, 705, 675. ${ }^{1} \mathrm{H}$ NMR (DMSO$\left.d_{6}\right): \delta 12.94$ (bs, $\left.1 \mathrm{H}, \mathrm{COOH}\right) ; 9.29(\mathrm{bs}, 1 \mathrm{H}, \mathrm{OH}), 8.40-8.46(\mathrm{~d}, 1 \mathrm{H}, J=8.0 \mathrm{~Hz}, \mathrm{NH}), 8.00$ (s, $1 \mathrm{H}, \mathrm{CHO}), 8.01$ (s, 2H, ArH), $7.06(\mathrm{~s}, 2 \mathrm{H}, \mathrm{ArH}), 4.53-4.56(\mathrm{~d}, J=5.6 \mathrm{~Hz}, 1 \mathrm{H}, \mathrm{CH}), 3.04-$ $3.10(\mathrm{dd}, J=9.2 \& 5.2 \mathrm{~Hz}, 1 \mathrm{H}, \mathrm{CH}), 2.81-2.89(\mathrm{dd}, J=14 \& 9.2 \mathrm{~Hz}, 1 \mathrm{H}, \mathrm{CH})$. Mass spectrum $m / z: 806[\mathrm{M}+\mathrm{H}]^{+}, 828[\mathrm{M}+\mathrm{Na}]^{+}, 844[\mathrm{M}+\mathrm{K}]^{+}$.

\section{Results and Discussion}

A thorough literature survey shows that there is no report is available for the synthesis of levothyroxine impurities such as $N$-methyl amide (2), T4-amine $O$-methyl (7), T4-acetamide (9) and $N$-formyl-T4 (11). These impurities including $N$-Acyl-T4 (10) are synthesized individually in pure form and well characterized based on their spectral data (IR, ${ }^{1} \mathrm{H}$ NMR and Mass). Further also confirmed by HPLC RRT as mention in USP.

Levothyroxine $N$-methyl amide (2) impurity prepared by reacting levothyroxine (1) with thionyl chloride in methanol to give corresponding methyl ester which on reacting with methylamine yields levothyroxine $N$-methyl amide or (S)-2-amino-3-(4-(4-hydroxy-3,5diiodophenoxy)-3,5-diiodophenyl)- $N$-methylpropanamide (2), Scheme 2.<smiles>N[C@@H](Cc1cc(I)c(Oc2cc(I)c(O)c(I)c2)c(I)c1)C(=O)O</smiles><smiles>CNC(=O)[C@H](N)Cc1cc(I)c(Oc2cc(I)c(O)c(I)c2)c(I)c1</smiles>

Scheme 2. Synthesis of levothyroxine $N$-methyl amide (2)

Preparation of levothyroxine T4-amine $O$-methyl (7) impurity is not reported but corresponding hydroxyl derivative is reported as multistep synthesis using aromatic boronic acid coupling as a key step ${ }^{7}$. To avoid this multistep synthesis and not readily available boronic 
acid intermediate prompted us to develop alternate synthesis method for impurity T4-amine $O$-methyl (7). Levothyroxine T4-amine $O$-methyl impurity i.e. 2-(4-(3,5-diiodo-4methoxyphenoxy)-3,5-diiodophenyl)ethanamine (7) was planned to synthesize from Boc protected levothyroxine including decarboxylation as key step. Decarboxylation of amino acid is reported using enzymes ${ }^{8}, 2$-cyclohexen-1-one ${ }^{9}$ catalyst and $N$-bromosuccinamide in buffer solution ${ }^{10}$.

Levothyroxine Boc protection was carried out as per literature procedure ${ }^{11}$ to yield $(S)-2$ ((tert-butoxycarbonyl)amino)-3-(4-(4-hydroxy-3,5-diiodophenoxy)-3,5-diiodophenyl) propanoic acid (3). (S)-Methyl 2-amino-3-(4-(3,5-diiodo-4-methoxyphenoxy)-3,5-diiodophenyl)propanoate (4) was obtained by reacting 3 with methyl iodide. Ester hydrolysis of $O$-methoxy ester (4) was achieved by base catalyst yields (S)-2-amino-3-(4-(3,5-diiodo-4-methoxyphenoxy)-3,5diiodophenyl)-propanoic acid (5) followed by acid catalyzed Boc deprotection using trifluoroacetic acid gives (S)-2-amino-3-(4-(3,5-diiodo-4-methoxyphenoxy)-3,5-diiodophenyl)propanoic acid (6) which on decarboxylation using catalytic amount of 2-cyclohexen-1-one in cyclohexanol gives T4-amine $O$-methyl (7) or 2-(4-(3,5-diiodo-4-methoxyphenoxy)-3,5diiodophenyl)ethanamine as mentioned in Scheme 3.
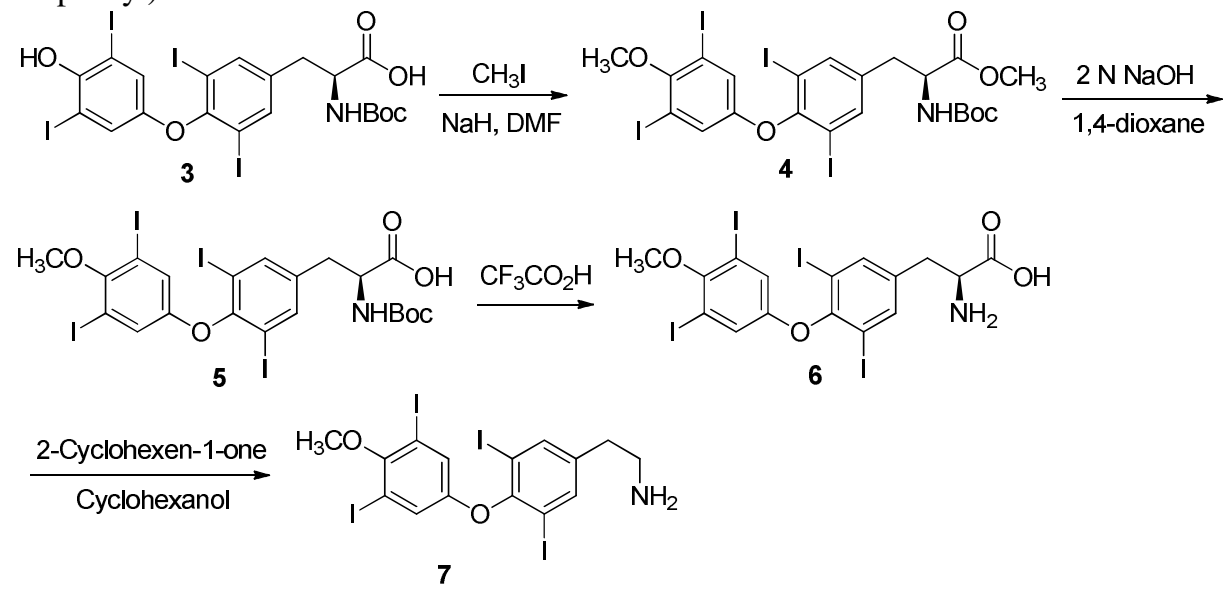

Scheme 3. Synthesis of T4-amine $O$-methyl (7)

T4-acetamide or 2-(4-(4-hydroxy-3,5-diiodophenoxy)-3,5-diiodophenyl)acetamide (9) impurity was obtained from commercially available corresponding acid i.e. 2-(4-(4-hydroxy3,5-diiodophenoxy)-3,5-diiodophenyl)acetic acid (8). T4-acetamide (9) was synthesized from acid (8) via acid chloride as a key intermediate obtained by reacting acid with thionyl chloride in chloroform, one pot amide was obtained by treating with ammonium hydroxide solution as in Scheme 4.<smiles>NC(=O)Cc1cc(I)c(Oc2cc(I)c(O)c(I)c2)c(I)c1</smiles>

Scheme 4. Synthesis of T4-acetamide (9)

$\mathrm{N}$-Acyl-T4 (10) and $\mathrm{N}$-formyl-T4 (11) impurities are prepared by acylation and formylation of levothyroxine (1) respectively. $\mathrm{N}$-Acylation of levothyroxine using acetic anhydride and triethylamine in $\mathrm{DMF}^{12}$ and use of $\mathrm{N}$-acetoxy siccinimide ${ }^{13}$ is already reported. Though detailed 
procedure was not reported using acetic anhydride and triethyl amine, we could not reproduce this protocol to get $N$-Acyl-T4 (10) with good purity. In this current procedure levothyroxine $N$-acylation was achieved using neat acetic anhydride to yield $N$-Acyl-T4 or (S)-2-acetamido-3-(4-(4-hydroxy-3,5-diiodophenoxy)-3,5-diiodophenyl)propanoic acid (10). $\mathrm{N}$-Formylation reaction was tried using formic acid and acetic anhydride as per reported procedure for another amino acid ${ }^{14} . N$-Formyl-T4 (11) impurity was synthesized by reacting levothyroxine (1) with formic acid in presence of acetic anhydride to give $N$-formyl-T4 or (S)-2-formamido-3-(4-(4-hydroxy-3,5-diiodophenoxy)-3,5-diiodophenyl)propanoic acid (11) as in Scheme 5.

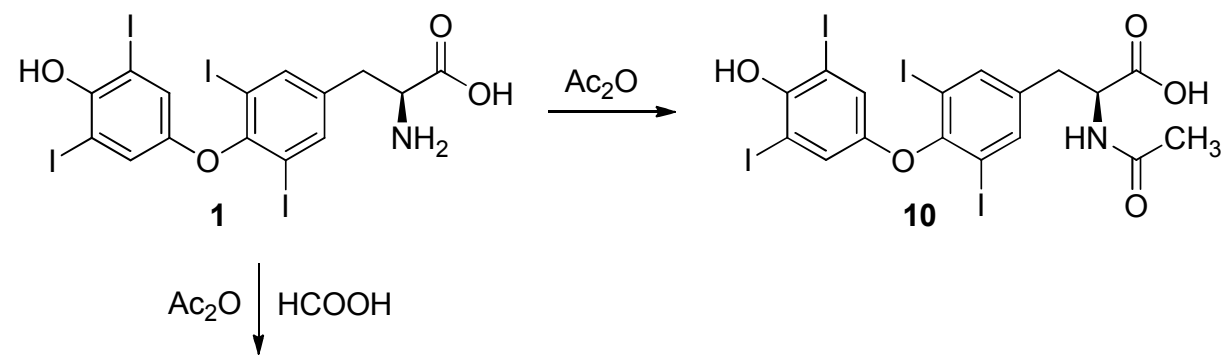<smiles>O=CN[C@@H](Cc1cc(I)c(Oc2cc(I)c(O)c(I)c2)c(I)c1)C(=O)O</smiles>

Scheme 5. Synthesis of $N$-acyl-T4 (10) and $N$-formyl-T4 (11)

\section{Conclusion}

Levothyroxine five potential impurities were synthesized individually either from levothyroxine drug or readily available raw material. These impurities were completely characterized by mass, IR and ${ }^{1} \mathrm{H}$ NMR.

\section{Acknowledgement}

The authors are thankful to the management of VerGo Pharma Research Lab. Pvt. Ltd. for providing facilities to carry out this study. Authors also express special thanks to Dr. Nitin Borkar (CEO) and Dr. S. K. Paknikar (Adviser) for their continuous encouragement for research work.

\section{References}

1. Harington C R, Biochem J., 1926, 20(2), 300-313; DOI:10.1042/bj0200300

2. Chalmers J R, Dickson G T, Elks J and Hems B A, J Chem Soc., 1949, 3424-3433; DOI:10.1039/JR9490003424

3. Khamar B M, Gurusamy R, Ravi M N, Reddy V M, Edde B, Ponnaiah R and Modi I A, Patent Appl No. WO2009136249.

4. $\quad$ Rao A P R and Dasari S, Patent Appl No. WO2015011573.

5. Chemburkar S R, Deming K C and Reddy R E, Tetrahedron, 2010, 66(11), 19551962; DOI:10.1016/j.tet.2009.12.044

6. Roy J, AAPS PharmSciTech, 2002, 3(2), 1-8; DOI:10.1208/pt030206 
7. Hart M E, Suchland K L, Miyakawa M, Bunzow J R, Grandy D K and Scanlan T S, $J$ Med Chem., 2006, 49(3), 1101-1112; DOI:10.1021/jm0505718

8. Hidetsugu N, Konosuke S, Keizo M and Koji M, Bioscience, Biotechnol Biochem., 1993, 57(7), 1210-1211; DOI:10.1271/bbb.57.1210

9. Hashimoto M, Eda Y, Osanai Y, Iwai T and Aoki S, Chem Lett., 1986, 15(6), 893896; DOI:10.1246/cl.1986.893

10. Laval G and Golding B T, Synlett, 2003, 4, 542-546.

11. Dorn A R, Salamone S J, Ghoshal M, Hoess E, Huber E, Hawley R C and Patterson J W, U. S. Patent 6,524,808 BI, 2003.

12. Davis P J and Mousa S A, US Patent No. 8802240 B2, 2014.

13. Navakouski M J, Vashkevich I I and Sviridov O V, Russian J Bioorg Chem., 2009, 35(2), 163-175; DOI:10.1134/S1068162009020046

14. Fayol A, Housseman C, Sun X, Janvier P, Bienaymé H and Zhu J, Synthesis, 2005, 1, 161-165; DOI:10.1055/s-2004-831225 